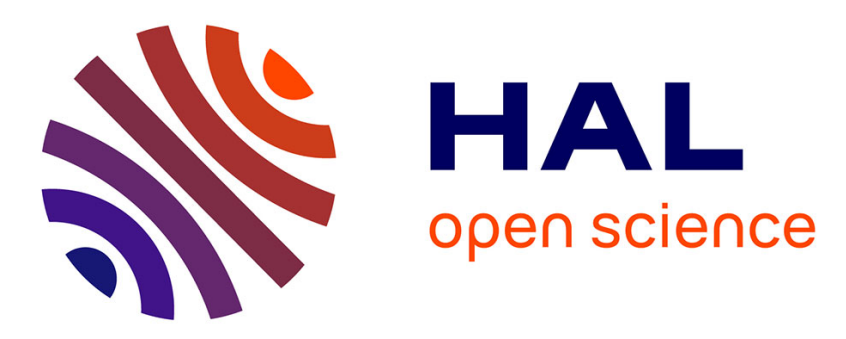

\title{
Melde's experiment on a vibrating liquid foam microchannel
}

Alexandre Cohen, Nathalie Fraysse, Christophe Raufaste

\section{To cite this version:}

Alexandre Cohen, Nathalie Fraysse, Christophe Raufaste. Melde's experiment on a vibrating liquid foam microchannel. Physical Review Letters, 2017, 119 (23), pp.238001. 10.1103/PhysRevLett.119.238001 . hal-01660212

\section{HAL Id: hal-01660212 https://hal.science/hal-01660212}

Submitted on 10 Dec 2017

HAL is a multi-disciplinary open access archive for the deposit and dissemination of scientific research documents, whether they are published or not. The documents may come from teaching and research institutions in France or abroad, or from public or private research centers.
L'archive ouverte pluridisciplinaire HAL, est destinée au dépôt et à la diffusion de documents scientifiques de niveau recherche, publiés ou non, émanant des établissements d'enseignement et de recherche français ou étrangers, des laboratoires publics ou privés. 


\title{
Melde's experiment on a vibrating liquid foam microchannel
}

\author{
Alexandre Cohen, ${ }^{1}$ Nathalie Fraysse, ${ }^{1}$ and Christophe Raufaste ${ }^{1, *}$ \\ ${ }^{1}$ Université Côte d'Azur, CNRS, Institut de Physique de Nice, 06100 Nice, France
}

(Dated: December 10, 2017)

\begin{abstract}
We subject a single Plateau border channel to a transverse harmonic excitation, in an experiment reminiscent of the historical one by Melde on vibrating strings, to study foam stability and wave properties. At low driving amplitudes, the liquid string exhibits regular oscillations. At large ones, a nonlinear regime appears and the acoustic radiation splits the channel into two zones of different cross section area, vibration amplitude, and phase difference with the neighboring soap films. The channel experiences an inertial dilatancy that is accounted for by a new Bernoulli-like relation.
\end{abstract}

Liquid foams have very specific and nonintuitive wave propagation properties due to their manifold structure made of gas bubbles, soap films and liquid microchannels, called Plateau borders (PBs) [1]. Their acoustic properties are not intermediate between the ones of their constitutive liquid and gas [2]. Foams can both act as metamaterials for given frequencies [3] and be used as efficient barriers against large amplitude or blast waves $[4,5]$. They also propagate shear waves in bulk $[6,7]$ or at the interfaces [8], and the existence of supershear Rayleigh waves has been proved following the impact of a projectile at high velocity [8]. The wave-propagation properties of liquid foams stem from the competition between an inertial contribution and an elastic one; however, they involve subtle local mechanisms that emphasize a coupling between soap films and PBs $[3,9,10]$. Investigations have been mainly performed in the linear regime and the response at large amplitudes remains very poorly studied and understood.

We reproduced Melde's experiment with a PB as the vibrating string, up to large amplitude oscillations. First, we show the existence of what we call an "inertial" dilatancy for the oscillating PB (see Refs. [11, 12] for the regular dilatancy effects in liquid foams). We derive a Bernoulli-like relation that accounts for the experimental data over almost four orders of magnitude: the larger its oscillation velocity, the smaller the pressure within the $\mathrm{PB}$ or, equivalently, the smaller the curvature radius of its cross section. Second, we show that the $\mathrm{PB}$ splits into two zones of different curvature radius and vibration amplitude above a critical forcing amplitude. We interpret this transition as a synchronization change between the coupled mechanical responses of the PB and of its holding films, in agreement with the measurements over three decades.

By pulling a triangular prism frame out of a surfactant solution, we create a horizontal PB and three soap films which span between the two solid triangular frame bases (Fig. 1a). The cross section of a PB consists in three arcs of circles in tangential contact; its transverse dimension is given by the radius of curvature of these arcs of circles, which we shorten in radius hereafter. The initial radius of

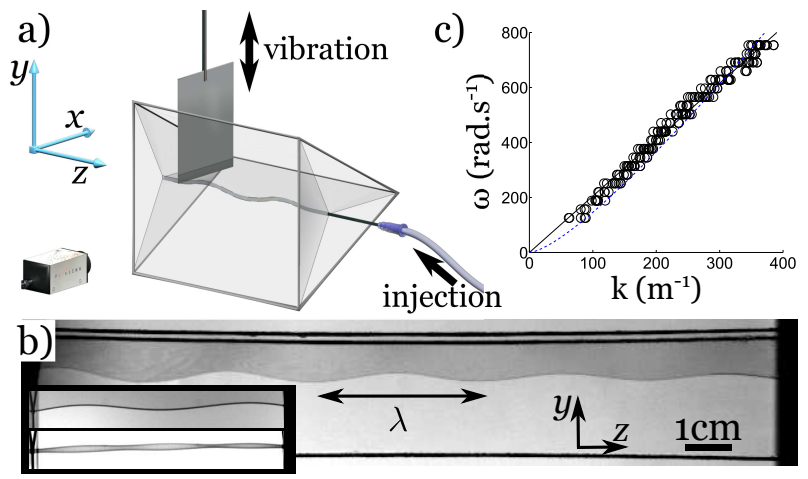

FIG. 1. a) Sketch of the experimental setup. b) Snapshot of an oscillating $15 \mathrm{~cm}$-long PB $(60 \mathrm{~Hz})$. Inset: A $5 \mathrm{~cm}$-long PB (top) and the envelope of the PB motion (bottom). c) Experimental dispersion relation for $R_{i}=0.1 \mathrm{~mm}$ and for several lengths of the PB (2 to $7 \mathrm{~cm})$ and widths of the films $(0.5,1.5$ and $3.3 \mathrm{~cm})$. The slope of the straight line is $c=2.0 \mathrm{~m} \cdot \mathrm{s}^{-1}$. The dashed blue line shows the result of the model of Derec et al. [14].

the $\mathrm{PB}, R_{i}$, is tuned by injecting liquid to compensate for the drainage [13]. It ranges between 0.1 and $1 \mathrm{~mm}$. We use a surfactant solution of TTAB (tetradecyl trimethyl ammonium bromide) at a concentration of $3 \mathrm{~g} \cdot \mathrm{l}^{-1}$ (density $\rho=1030 \mathrm{~kg} \cdot \mathrm{m}^{-3}$, surface tension $\gamma=36 \mathrm{mN} \cdot \mathrm{m}^{-1}$ and dynamic viscosity $\eta=1.04 \mathrm{mPa} \cdot \mathrm{s}[13])$.

We subject the system to a mechanical excitation by means of a mobile plate (Fig. 1a) rigidly connected to the axis of a vibrator, which imposes a sinusoidal and transverse motion in the frequency range $30-120 \mathrm{~Hz}$. The time scale of the soap film drainage is larger than $1 \mathrm{~s}$, which makes it possible to investigate the response of the system to the harmonic excitation on intermediate time scales $(\sim 100 \mathrm{~ms})$ much larger than the period of vibration $(\sim 10 \mathrm{~ms})$, while neglecting gravity. The PB dynamics is recorded using a high-speed camera (1000 to $5000 \mathrm{fps}$ ). The excitation generates a transverse wave along the PB in the zy-plane (Fig. 1b). When the mobile plate contacts the whole system made of the $\mathrm{PB}$ and its three holding films, the PB exhibits an elliptic trajectory in any xy-plane, whereas this trajectory is linear, along the $y$-axis, when the mobile plate contacts the horizontal 

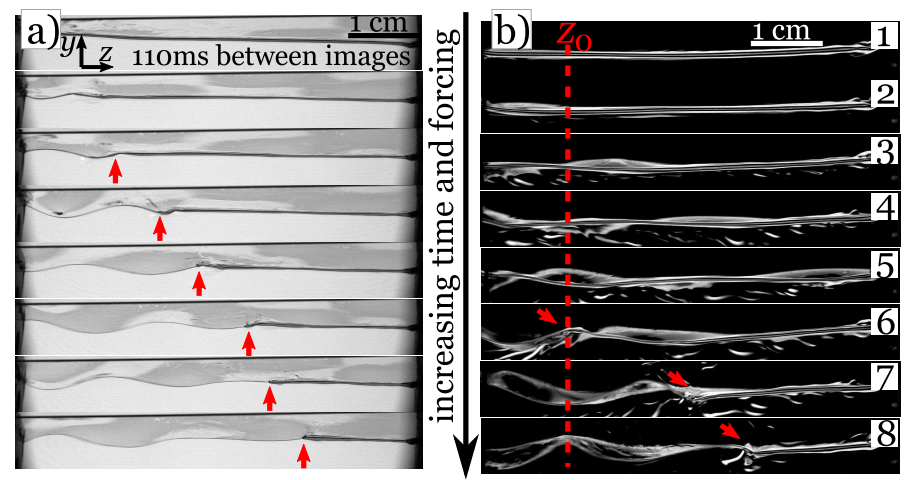

FIG. 2. Image sequence obtained at increasing driving amplitude. The red arrows emphasize the position of the front. a) Bright field imaging $(60 \mathrm{~Hz})$. b) Dark field imaging $(40 \mathrm{~Hz})$. The red dashed line indicates $z_{0}$, the position selected to plot the space-time diagram of Fig. 3.

film only, as in Fig. 1a. We checked that similar features are observed in both cases and retained the second configuration for simplicity. The time average of the image sequence shows the envelope of the PB motion and reveals the presence of nodes and antinodes at all frequencies. The nodes amplitude is not exactly zero and the waves are not pure standing waves.

Fig. 1c displays the experimental dispersion relation $\omega=2 \pi f$ vs $k=2 \pi / \lambda$, where $\lambda$ is the wavelength, obtained at small amplitude for various lengths of the PB and various widths of the holding films. The dispersion relation is consistent with the expression derived by Derec et al. in the range $20-2000 \mathrm{~Hz}$ [9], which predicts that the oscillations of the soap films, including the effect of the air they set into motion, dominate at low frequency, whereas the inertia of the $\mathrm{PB}$ prevails at high frequency. Most of our measurements are dominated by the oscillation of the soap films [14]. In practice, a nondispersive dispersion relation remains a good approximation to describe the small amplitude regime (Fig. 1c). The best linear adjustment yields a value of the phase velocity $c=2.0 \pm 0.1 \mathrm{~m} \cdot \mathrm{s}^{-1}$. Attenuation becomes significant when the PB length reaches $15 \mathrm{~cm}$ (Fig. 1b). In what follows, we will set the length of the $\mathrm{PB}$ at $5 \mathrm{~cm}$, and the width of the films at $1.5 \mathrm{~cm}$.

At large amplitude excitation, the system undergoes a transition toward a highly nonlinear regime. The PB splits into two distinctive zones as illustrated in Fig. 2. On the side of the moving plate, the PB appears to be thin and undergoes large-amplitude oscillations. Conversely, on the side of the fixed injection plate, the PB is thick and hardly vibrates. In between these two zones, hereafter, respectively, referred to as the "thin" and "thick" zones, a transition region a few millimeters long displays a rather sharp jump in $\mathrm{PB}$ radius, referred to as the "front". This region is also characterized by a peri-

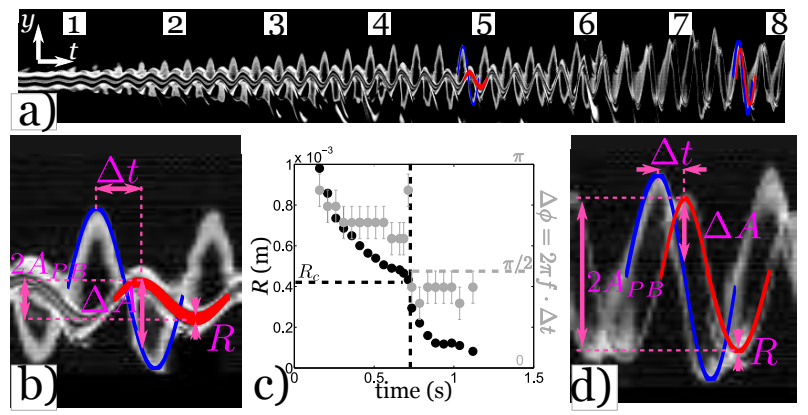

FIG. 3. a) Space-time diagram for the experiment reported in Fig. 2b. Labels (1) to (8) indicate the time positions of the numbered images of Fig. $2 \mathrm{~b}$. The oscillations of both the PB and the film are visible. The passage of the front at $z_{0}$ occurs for time label (6). b) Enlargement prior to time label (6). The $\mathrm{PB}$ and the envelope of the film are outlined in blue and red, respectively, over one oscillation time period. c) PB radius $R$ (black circles) and phase difference $\Delta \phi=2 \pi f \Delta t$ (light gray circles) as functions of time. The passage of the front occurs for $R_{c}=0.42 \mathrm{~mm}$. d) Same as b) after time label (6).

odic discharge of liquid from the PB to the holding soap films. We observe that the larger the driving amplitude, the longer the thin zone. No significant hysteresis was observed when decreasing the amplitude of excitation. The process is fully reversible and the linear regime is retrieved at small amplitude.

Quantitative measurements are performed by imaging both the PB and the horizontal film in the background using a "dark field" configuration obtained by screening the direct light beam. The PB appears as a dark-brightdark stripe, while the envelope of the soap film projection in the zy-plane is homogeneously bright (Fig. 2b). We now impose a sweep in driving amplitude at a typical rate of $10 \mathrm{~mm} \cdot \mathrm{s}^{-1}$. This is slow enough to consider that the driving amplitude is constant over a time period; we checked that the results do not depend on this rate. For each experiment, we build a space-time diagram along a line $z=z_{0}$, with $z_{0}$ at the first antinode of the linear regime to maximize the signal-to-noise ratio. Note that the following results do not depend on the choice of the antinode. Fig. 3a gives an example obtained from the experiment of Fig. 2b. Various local quantities are measured for each period: the oscillation amplitude of the $\mathrm{PB}, A_{P B}$ (defined from rest to crest), the $\mathrm{PB}$ radius, $R$, the difference in amplitude of oscillation between the $\mathrm{PB}$ and the film, $\Delta A$ (measured when the $\mathrm{PB}$ position is maximal), and the phase difference between the film oscillation and the PB oscillation, $\Delta \phi$, where $\Delta \phi=2 \pi f \Delta t$ and $\Delta t$ is the time delay between the film and the PB.

We observe that the PB and film amplitudes first increase linearly with the driving amplitude. A sudden increase in the amplitude of the $\mathrm{PB}$, concomitant to a rapid decrease in the $\mathrm{PB}$ radius (Fig. 3c), marks the passage of the front at $z_{0}$. The film and the PB oscillate ap- 

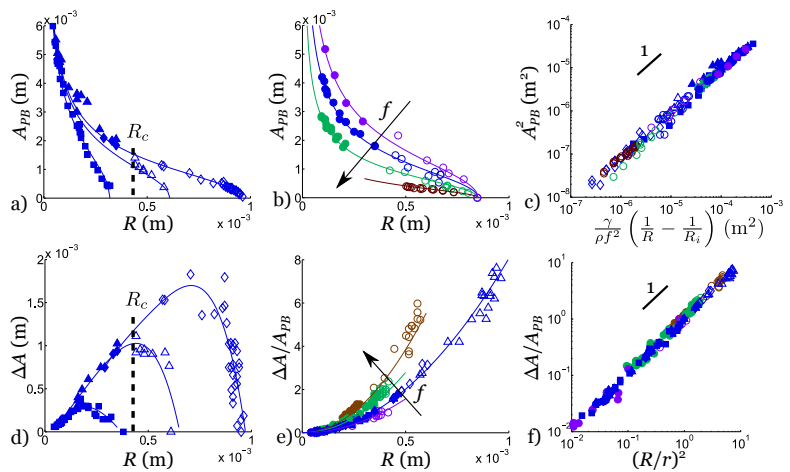

FIG. 4. (a-c) Results on the amplitude $A_{P B}$ of the PB. The solid lines are given by Eq. 2 with 0.084 as the proportionality factor. (a) $A_{P B}$ as a function of $R$ for a frequency $f=40 \mathrm{~Hz}$ and various initial radii $R_{i}(0.32(\square), 0.61(\triangle)$ and $0.96(\diamond) \mathrm{mm})$. The vertical dashed line pinpoints the critical radius $R_{c}=0.42 \mathrm{~mm}$. (b) $A_{P B}$ as a function of $R$ for an initial radius $R_{i}=0.85 \mathrm{~mm}$ and various frequencies (30, 40,60 and $120 \mathrm{~Hz}$ ). (c) $A_{P B}^{2}$ as a function of $\frac{\gamma}{\rho f^{2}}\left(\frac{1}{R}-\frac{1}{R_{i}}\right)$ (log-log scale). (d-f) Results on the amplitude difference $\Delta A$. (d) $\Delta A$ as a function of $R$ for the same experiments as in (a). $R_{c}=0.42 \mathrm{~mm}$ is represented. The solid lines are guides for the eyes. (e) $\Delta A / A_{P B}$ as a function of $R$. Solid lines are parabolic fits, $(R / r(f))^{2}$, where $r$ is a free parameter adjusted for each frequency $\left(30,40,60\right.$ and $80 \mathrm{~Hz}$ ). (f) $\Delta A / A_{P B}$ as a function of $(R / r(f))^{2}$ (log-log scale).

proximately out of phase before the passage of the front $(\Delta \phi>\pi / 2)$, that is to say in the thick zone, whereas they oscillate approximately in phase after the passage of the front $(\Delta \phi<\pi / 2)$, in the thin zone (Fig. 3c). We note $R_{c}$ the critical radius that marks the passage of the front. The critical radii are close to $0.4 \mathrm{~mm}$ in all our measurements. $R_{c}$ does not significantly depend on the initial PB radius $R_{i}$, but slightly decreases when the driving frequency increases (Fig. 5b). The best adjustment of the data leads to the following relationship:

$$
R_{c}(m)=(2.1 \pm 0.3) \cdot 10^{-3} f(H z)^{-0.41 \pm 0.04}
$$

Fig. 4 summarizes the measurements made on space-time diagrams for experiments performed during successively increasing and decreasing ramps in amplitude of excitation.

The amplitude of the PB appears to be a decreasing function of its radius at given frequency $f$ and initial $\mathrm{PB}$ radius $R_{i}$ (Figs. 4a,b). As already mentioned, the thicker the $\mathrm{PB}$, the smaller its oscillations. A slight decrease in the PB amplitude is observed at increasing frequencies for a given $R_{i}$, or at decreasing $R_{i}$ for a given frequency. Empty and solid symbols were used in Fig. 4 to differentiate the data obtained before and after the front reaches $z_{0}$, respectively. Strikingly, the experimental curves of Figs. 4a,b are continuous and smooth; no particular feature of the curves reveals the passage of the front.
Given a typical velocity scale $A_{P B} f$ with $A_{P B} \sim 4 \mathrm{~mm}$ and $f \sim 50 \mathrm{~Hz}$, we can build a Reynolds number $R e=$ $\rho R A_{P B} f / \eta$ for the velocity variations inside the PB. With $R \sim 0.5 \mathrm{~mm}$, we get $R e \sim 100$, which calls for an inertial regime. Taking $\lambda(1.7-6.7 \mathrm{~cm}$ in this study) instead of $R$ for the length scale leads to a higher value and the same conclusion holds. The existence of inertial flows inside PBs has recently been proved and the coupling between the capillary and inertial effects shown to exhibit highly nonlinear features such as the propagation of hydraulic jumps and solitons [15-17]. Assuming a capillaryinertial mechanism, we write a Bernoulli-like relation by balancing a typical kinetic energy per volume $\rho\left(A_{P B} f\right)^{2}$ and the pressure difference $P_{i}-P=\gamma\left(\frac{1}{R}-\frac{1}{R_{i}}\right)$ given by the Laplace formula (note that the sign (-) is due to the concave free surface of the PB cross section):

$$
A_{P B}^{2} \sim \frac{\gamma}{\rho f^{2}}\left(\frac{1}{R}-\frac{1}{R_{i}}\right)
$$

This scaling was tested on our measurements. Fig. 4c shows a remarkably good fit with all the experimental data over almost four orders of magnitude; the best adjustment yields a proportionality factor of $0.084 \pm 0.003$. Eq. 2 proves to capture all the fine details of the trends observed in Fig. 4a,b. The relation shows that the PB is dilatant and that the larger its oscillation velocity, the smaller its radius. Note that Eq. 2 expresses an intimate signature of the PB internal flow and geometry, without any reference to the coupling of the $\mathrm{PB}$ with the holding films. We thus do not expect this local relationship to provide any sign of the passage of the front, in agreement with our observations.

The difference in amplitude $\Delta A$ connects to the coupling between the soap film and the PB. Measurements at a given frequency and various initial radii lead to bellshaped curves that all collapse to a unique curve, monotonically increasing with the $\mathrm{PB}$ radius, when $\Delta A$ is normalized by the PB amplitude, $A_{P B}$. As an example, all the data of Fig. 4d collapse in Fig. 4e (dark blue symbols) and can be fitted by a parabola (dark blue line) over three orders of magnitude by introducing the fitting length $r$ :

$$
\frac{\Delta A}{A_{P B}}=\left(\frac{R}{r(f)}\right)^{2}
$$

Again, no particular feature on the curves reveals the passage of the front. All the data obtained for various frequencies collapse on the same curve (Fig. 4f) with $r$ values slightly decreasing with $f$ (Fig. 5b):

$$
r(m)=(1.9 \pm 0.1) \cdot 10^{-3} f(H z)^{-0.46 \pm 0.02}
$$

Models of the coupling between a PB and its holding films have been derived by Seiwert et al. [10] for their particular circular geometry, and by Derec et al. [9] for 

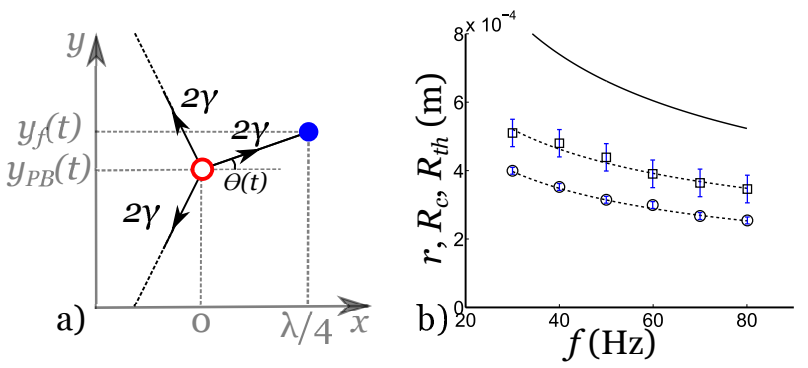

FIG. 5. a) Sketch of the model. The PB is symbolized by the red empty spot. It is driven by the horizontal soap film, whose maximum in the xy-plane is displayed by the blue solid spot. Each film pulls on the PB with a force $2 \gamma$ per unit length. b) Measurements of $R_{c}(\square)$ and $r(\circ)$ and their interpolations (dashed lines) given by Eqs. 1 and 4 . The model $R_{t h}$ is displayed by the solid line.

small-amplitude excitations. Here we demonstrate that a simple model of driven oscillator having a PB radiusdependent eigenfrequency successfully describes the coupling between the PB and the films, as well as the oscillatory response of the $\mathrm{PB}$ when combined with the dilatancy effect, which links the radius and amplitude of the PB (Eq. 2).

Each section of the PB (xy-plane) is considered as independent and driven by the motion of the soap films in its vicinity, leading to the $2 \mathrm{D}$ problem sketched in Fig. $5 \mathrm{a}$. The force exerted by the horizontal film is assumed to point toward the first maximum of the film envelope in the xy-plane; this defines the angle $\theta$. The PB is assumed to be located close to a node of vibration of the film. This assumption is checked experimentally at least in the thick zone where the amplitude of the PB is significantly smaller than the one of the horizontal film. In consequence, the distance between the $\mathrm{PB}$ and the film maximum is approximately equal to a quarter of the wavelength, $\lambda / 4$, and $\theta(t) \sim \frac{y_{f}(t)-y_{P_{B}}(t)}{\lambda / 4}$ in the limit of small angles, where $y_{f}$ and $y_{P B}$ are the y-positions of the film maximum and of the $\mathrm{PB}$, respectively. At the 1st order in $\theta$, the forces exerted on the PB by its three holding films compensate in the $\mathrm{x}$-direction, but not in the $\mathrm{y}$-direction, where the force equals $2 \gamma \theta$. The momentum per unit length equation is $\rho S \frac{d^{2} y_{P B}(t)}{d t^{2}}=\frac{8 \gamma}{\lambda}\left(y_{f}(t)-y_{P B}(t)\right)$, where $S=(\sqrt{3}-\pi / 2) R^{2}$ is the area of the PB cross section [9] and $\lambda=c / f$ with $c$ taken equal to $2 \mathrm{~m} \cdot \mathrm{s}^{-1}$. The equation of motion of each cross section of the $\mathrm{PB}$ can thus be rewritten into the form of the differential equation of a driven linear oscillator:

$\frac{d^{2} y_{P B}(t)}{d t^{2}}+\frac{2 \pi f_{0}}{Q} \frac{d y_{P B}(t)}{d t}+\left(2 \pi f_{0}\right)^{2} y_{P B}(t)=\left(2 \pi f_{0}\right)^{2} y_{f}(t)$

where the eigenfrequency $f_{0}=\sqrt{\frac{2 \gamma}{\pi^{2} \rho S \lambda}}=$ $\sqrt{\frac{2}{(\sqrt{3}-\pi / 2) \pi^{2}} \frac{\gamma f}{\rho R^{2} c}}$ depends on the local PB radius
$R$. A linear damping term can be included through the use of a quality factor $Q$. We do not discuss its physical meaning since all the following results do not depend on $Q$. We note $y_{f}(t)=A_{f} \cos (2 \pi f t)$ the harmonic motion of the film envelope and we look for the steady state harmonic solution of the $\mathrm{PB}$ motion under the form $y_{P B}(t)=A_{P B} \cos (2 \pi f t-\Delta \phi)$. Classically, the amplitude and phase are given by $A_{P B}=\frac{A_{f}}{\sqrt{\left(1-\left(\frac{f}{f_{0}}\right)^{2}\right)^{2}+\left(\frac{f}{f_{0} Q}\right)^{2}}}$ and $\cos (\Delta \phi)=\frac{1-\left(\frac{f}{f_{0}}\right)^{2}}{\sqrt{\left(1-\left(\frac{f}{f_{0}}\right)^{2}\right)^{2}+\left(\frac{f}{f_{0} Q}\right)^{2}}}$. The model predicts a phase change for $f_{0}=f$ or, equivalently, for $R=R_{t h}$ where

$$
R_{t h} \simeq 1.12 \cdot\left(\frac{\gamma}{\rho c}\right)^{1 / 2} \cdot f^{-1 / 2},
$$

or $R_{t h}(m) \simeq 4.69 \cdot 10^{-3} \cdot f(H z)^{-1 / 2}$ with our parameters (Fig $5 \mathrm{~b}$ ). For large radii of the $\mathrm{PB}, R>R_{t h}$, the $\mathrm{PB}$ and the film oscillate out of phase $(\Delta \phi>\pi / 2)$. Conversely, the thin zones of the PB and the film vibrate in phase as $R<R_{t h} . R_{t h}$ is thus a prediction of the critical radius $R_{c}$ measured experimentally. The predicted exponent $-1 / 2$ for the frequency dependence is consistent with the measurements and there is less than a factor 2 on the prefactors (Eq.1), which is remarkable considering the simplicity of the model. Regarding $\triangle A$, the model gives $\Delta A=A_{P B}-A_{f} \cos (\Delta \phi)$, which leads to $\Delta A / A_{P B}=\left(\frac{f}{f_{0}}\right)^{2}=\left(\frac{R}{R_{t h}}\right)^{2}$ after calculations. The model retrieves the experimental parabolic behavior and $R_{t h}$ can also be assimilated to $r$. Again, the agreement with the experimental data (Eq. 4) is very good (Fig. 5b).

Combining this model and the inertial dilatancy effect for the oscillating PB, we can elaborate the following scenario for an experiment performed at a given frequency and increasing driving amplitude. As long as the amplitude of oscillation remains small, the $\mathrm{PB}$, which we assume initially uniform and rather thick, oscillates out of phase with the films and the eigenfrequency of the oscillator corresponding to any cross section of the system is smaller than the driving frequency. As the amplitude of the mobile plate increases, the amplitude of the $\mathrm{PB}$ increases as well; due to the inertial dilatancy characterized by the Bernoulli-like relationship of Eq. 2, the radius of the PB decreases, which in turn leads to an increase in the oscillator eigenfrequency. Assuming that the oscillation amplitude for the $\mathrm{PB}$ is the largest close to the mobile plate, we expect that the oscillator eigenfrequency reaches the value of the driving frequency there first; simultaneously, a change in phase between the PB and the films occurs. As the driving amplitude increases further, the oscillator eigenfrequency becomes larger than the driving frequency over a more and more extended zone, starting at the mobile plate and ending for a critical $\mathrm{PB}$ radius, or, equivalently, a critical $\mathrm{PB}$ oscillation am- 
plitude. This leads to the experimentally observed drift of a front that separates the out of phase / thick zone from the in phase / thin zone, toward the fixed base.

The present study emphasizes the subtle coupling that exists between the basic constituents of liquid foams and brings to light a new inertial dilatancy effect of their Plateau borders. Large amplitude perturbations lead to a nonlinear regime that has direct consequences for foam stability and wave properties.

We thank C. Derec, F. Elias, B. Dollet, and J. Rajchenbach for fruitful discussions and B. Gay-Para for the sketch of the setup.

* corresponding author: Christophe.Raufaste@unice.fr

[1] I. Cantat et al, Foams. Structure and Dynamics (edited by S. Cox, Oxford University Press 2013).

[2] A. B. Wood, A Textbook of Sound (Bell, London, 1944).

[3] J. Pierre, B. Dollet, and V. Leroy, Phys. Rev. Lett. 112, 148307 (2014).

[4] I. Goldfarb, Z. Orenbakh, I. Shreiber, and F. Vafina, Shock Waves 7, 77-88 (1997).

[5] M. Monloubou, A. Saint-Jalmes, B. Dollet, and I. Cantat, EPL 112, 34001 (2015).

[6] Q. Sun, J. P. Butler, B. Suki, and D. Stamenović, J. Colloid Interface Sci. 163, 269-276 (1994).

[7] F. Wintzenrieth, S. Cohen-Addad, M. Le Merrer, and R. Höhler, Phys. Rev. E 89, 012308 (2014).

[8] A. Le Goff, P. Cobelli, and G. Lagubeau, Phys. Rev. Lett. 110, 236101 (2013).

[9] C. Derec,V. Leroy ,D.Kaurin, L. Arbogast, C. Gay, and F. Elias EPL 112, 34004 (2015).

[10] J. Seiwert, J. Pierre, and B. Dollet, J. Fluid Mech. 788, 183-208 (2016).

[11] D. Weaire and S. Hutzler, Phil. mag. 83, 2747-2760 (2003)

[12] S. P. L. Marze, A. Saint-Jalmes, and D. Langevin, Colloids Surf. A 263, 121-128 (2005).

[13] A. Cohen, N. Fraysse, and C. Raufaste, Phys. Rev. E 91, 053008 (2015).

[14] The expression derived by Derec et al. [9] gives $k=$ $\sqrt{\left(\frac{\rho_{a}}{\gamma}\right)^{2 / 3} \omega^{4 / 3}+\frac{\rho e}{3 \gamma} \omega^{2}+\left(\frac{0.161 \rho R^{2}}{3 \gamma}\right)^{2} \omega^{4}}$, where $\rho_{a}$ is the air density and $e$ is the thickness of the soap films. Fig. 1c shows a good agreement between the data and the above theoretical expression using values of $e=10 \mu \mathrm{m}$ for the film thickness and $R=100 \mu \mathrm{m}$ for the PB radius. Under our experimental conditions, the dispersion relation is dominated by the oscillation of the soap films, including the effect of the air they set into motion, in agreement with the criterion $f \cdot R^{3 / 2}<46 \mathrm{~mm}^{3 / 2}$. $\mathrm{s}^{-1}$ [9]. Only the thickest PBs do not fulfill this criterion and the inertia of the PB (3rd term under the square root) should not be neglected for them.

[15] A. Cohen, N. Fraysse, J. Rajchenbach, M. Argentina, Y. Bouret, and C. Raufaste, Phys. Rev. Lett. 112, 218303 (2014).

[16] M. Argentina, A. Cohen, Y. Bouret, N. Fraysse, and C.
Raufaste, J. Fluid Mech. 765, 1-16 (2015).

[17] Y. Bouret, A. Cohen, N. Fraysse, M. Argentina, and C.Raufaste, Phys. Rev. F 1, 043902 (2016). 